\title{
Reference change value and measurement uncertainty of CRP, ferritin, procalcitonin, troponin I, and BNP tests in COVID-19 monitoring: How much change is significant?
}

\author{
Nergiz Zorbozan', (D) Orcun Zorbozan² \\ 'Department of Biochemistry, Izmir Kemalpasa State Hospital, Izmir, Turkey \\ ${ }^{2}$ Department of Medical Parasitology, Ege University Faculty of Medicine, Izmir, Turkey
}

\begin{abstract}
Objectives: This study was designed to determine the point of clinically significant change in consecutive measurements of high concentrations of C-reactive protein (CRP), ferritin, procalcitonin, troponin I, and brain natriuretic peptide (BNP) in patients diagnosed with COVID-19.

Methods: The reference change value (RCV) for each analyte was estimated using the following formula: $R C V=2^{1 /}$ ${ }^{2} \times \mathrm{Z} \times\left(\mathrm{CV}_{\mathrm{a}}{ }^{2}+\mathrm{CV}_{\mathrm{i}}\right)^{1 / 2}$. The combined RCV was calculated using the mean $\mathrm{CV}$ value determined using different concentrations. Measurement uncertainty (MU) was estimated according to NordTest guideline. The root-mean-square (RMS) bias and nominal uncertainty values (uCref) were calculated. Standard uncertainty (ubias) and combined standard uncertainty $(\mathrm{u})$ were calculated using the formula RMSbias ${ }^{2}+\mathrm{uCref}^{2}$ and $\sqrt{ }\left(\mathrm{uRW}^{2}+\mathrm{ubias}^{2}\right) / 2$, respectively. Expanded uncertainty (U) was obtained by multiplying u by a coverage factor.

Results: The lowest RCV values of CRP, ferritin, procalcitonin, BNP, and troponin I tests were $94.57 \%, 37.16 \%, 40.55 \%$, $42.01 \%$, and $28.99 \%$, respectively. The combined RCV value was $94.6 \%, 38.31 \%, 41.57 \%, 42.35 \%$, and $29.36 \%$ for CRP, ferritin, procalcitonin, troponin I, and BNP, respectively. The U of the CRP test was between $4.70 \%$, and $5.49 \%$. The lowest MU values of ferritin, troponin I, and BNP tests were $48.45 \%, 48.24 \%$, and $53 \%$, respectively.

Conclusion: Providing the RCV and MU values with the test results in laboratory reports of these important biomarkers, which are frequently seen in high concentrations in COVID-19 patients, would offer valuable information for more accurate clinical interpretation.
\end{abstract}

Keywords: COVID-19, measurement uncertainty, reference change value

C oronavirus disease 2019 (COVID-19) can have neurological, cardiovascular, and other end-organ involvement, in addition to isolated lung disease. The early recognition of the severity of COVID-19 and timely triage of patients has become even more important due to the large number of patients and difficulties providing treatment in intensive care units.

C-reactive protein (CRP), ferritin, procalcitonin, troponin I, and brain natriuretic peptide (BNP) tests are biomarkers that are known to increase in patients with COVID-19 due to inflammatory response and disease involvement. Changes in blood concentrations of these biomarkers are used as predictors of clinical severity and complications.
The determination of biomarker levels and monitoring them with sequential measurements is used in the follow-up and treatment of patients who are undergoing long-term intensive care treatment or are at risk of death [1-4]. Therefore, the correct interpretation of the test results reported by laboratories is essential. Clinicians often use tools like clinical decision points and biological reference values to interpret patient test results [5].

Laboratories check the analytical variation of the tests for the levels at clinical decision points and biological reference limits using an internal quality control sample. Since the onset of the COVID-19 pandemic, clinicians and laboratory profession-

Address for correspondence: Nergiz Zorbozan, MD. Department of Biochemistry, Izmir Kemalpasa State Hospital, Izmir, Turkey Phone: +90 5443213469 E-mail: nergiz_girgin@hotmail.com ORCID: 0000-0001-7298-1897

Submitted Date: June 11, 2021 Accepted Date: August 14, 2021 Available Online Date: September 14, 2021

${ }^{\circ}$ Copyright 2021 by International Journal of Medical Biochemistry - Available online at www.internationalbiochemistry.com OPEN ACCESS This work is licensed under a Creative Commons Attribution-NonCommercial 4.0 International License. 
als have faced a new challenge. Laboratories have added new tests to their test panels due to new clinical needs. In addition, the levels of CRP, ferritin, procalcitonin, troponin I, and BNP in COVID-19 patients are often at concentrations much higher than the clinical decision limits and population-based reference ranges $[1,2]$. Therefore, laboratories should more strictly control analytical variations at high concentrations in addition to known clinical decision limits. Similarly, it is important to determine the clinical significance of the change between 2 consecutive measurement results since the patients are often followed up with sequential measurements of these analytes [1-4].

Harris and Brown [6] were the first authors to objectively interpret the change between 2 consecutive values while measuring the same analyte at different times in a single individual. They reported that the difference can be associated with the change in the health status of the patient when the variation between 2 consecutive values is greater than the intra-individual biological variability and analytical variation. They defined the concept of reference change value (RCV), which defines the minimal significant difference between measurements performed at different times [6, 7].

Measurement uncertainty (MU) refers to the statistical distribution of the values attributed to a measured quantity. It is a value that expresses a lack of knowledge about the true value of a test result. This is not an indication of the error in the measuring system, but an inevitable natural feature of the variability of measurement conditions. The result of any measurement represents an approximation or estimate of the value of the measured quantity. Therefore, additional information about the uncertainty of the estimate adds value to a test result [8].

The objective of this study was to determine the percentage of clinically significant change and the MU values of the change for consecutive measurement results of high concentrations of CRP, ferritin, procalcitonin, troponin I, and BNP in COVID-19 patients.

\section{Materials and Methods}

The ferritin, procalcitonin, troponin I, and BNP tests were performed using an Advia Centaur XP Immunoassay System (Siemens Healthineers $\mathrm{GmbH}$, Erlangen, Germany). The procalcitonin measurement method is a sandwich immunoassay containing a fluorescein monoclonal antibody. Ferritin, troponin I, and BNP tests are measured using the chemiluminometric sandwich immunotest method. CRP testing was performed using the Advia 1800 clinical chemistry analyzer (Siemens Healthineers $\mathrm{GmbH}$, Erlangen, Germany) using a latex-enhanced immunoturbidimetric method. Plasma and serum sample pools were prepared to determine the analytical variation values of the test. The plasma pool was used for the BNP test and the serum pool for the remaining tests. Sample pool concentrations were determined according to the sample concentrations of CRP, ferritin, procalcitonin, troponin I, and BNP of patients hospitalized with a diagnosis of COVID-19 and clinical decision limits. The sample pools were prepared using the remaining routine samples without lipemia, icterus, or hemolysis. Samples were aliquoted and stored at $2-8^{\circ} \mathrm{C}$ for 1 month until analysis. Prior to beginning the analysis, the samples were allowed to reach room temperature and were vortexed. Sample pools were consecutively assayed 20 times. All of the samples were measured using a single calibration curve and a single reagent lot was used during the experiment. Normal quality control procedures were performed during the analytical coefficient of variation $\left(\mathrm{CV}_{\mathrm{a}}\right)$ experiment. The coefficient of variation (CV) was calculated using the formula of SDx100/laboratory mean of serum pool.

Intra-individual coefficient of variation $\left(\mathrm{CV}_{\mathrm{i}}\right)$ values of $34.1 \%$ and $12.8 \%$ were obtained for CRP and ferritin, respectively, from the European Federation of Clinical Chemistry and Laboratory Medicine (EFLM) Biological Variation Database managed by the EFLM Biological Variation Working Group and the Biological Variation Database Task Group [9]. The $\mathrm{CV}_{i}$ value of procalcitonin was $14 \%[10]$, and the $\mathrm{CV}_{\mathrm{i}}$ value of troponin I and BNP was $14.05 \%$ and $10 \%$, respectively [11] (Table 1).

The RCV for each analyte was estimated using the following formula: $\mathrm{RCV}=2^{1 / 2} \times \mathrm{Z} \times\left(\mathrm{CV}_{\mathrm{a}}{ }^{2}+\mathrm{CV}_{i}{ }^{2}\right)^{1 / 2}$. Two-sided Z-scores were used since the decrease and increase in consecutive measurements of analyte concentrations was important in clinical decision-making (1.96 for $p<0.05$ and 2.58 for $p<0.01)$ [12].The combined RCV value for each test was calculated using the mean $\mathrm{CV}$ value calculated for different concentrations.

$\mathrm{MU}$ was estimated using the top-down method according to the NordTest guideline [13]. The CRP, ferritin, troponin I, and BNP measurement procedures performed in our laboratory are registered with the Clinical Chemistry External Quality Assurance Services (EQAS) Bio-Rad program. External quality control of the tests is performed monthly. For the MU estimation of the tests,

Table 1. Intra-individual coefficient of variation data of CRP, ferritin, procalcitonin, troponin I, and BNP tests

\begin{tabular}{llllr}
\hline Analyte & Sample type & BV estimate & Median CV estimate (\%) \\
\hline CRP $(\mathrm{mg} / \mathrm{L})$ & Serum/plasma & Within-subject & $34.1^{\mathrm{a}}$ & Date \\
Ferritin $(\mathrm{ug} / \mathrm{L})$ & Serum/plasma & Within-subject & $12.8^{\mathrm{a}}$ & 2020 \\
Procalcitonin $(\mathrm{ng} / \mathrm{mL})$ & Serum & Within-subject & $14^{\mathrm{b}}$ & 2020 \\
Troponin I $(\mathrm{ng} / \mathrm{L})$ & Serum & Within-subject & $14.05^{\mathrm{c}}$ & 2004 \\
BNP $(\mathrm{pg} / \mathrm{mL})$ & Serum & Within-subject & $10^{c}$ & 2014 \\
\hline
\end{tabular}

a: Derived from the European Federation of Clinical Chemistry and Laboratory Medicine biological variation database (9). b: Derived from Barassi et al. study data (10). c: Derived from the Westgard online database (11). BNP: Brain natriuretic peptide; BV: Biological variation; CRP: C-reactive protein; CV: Coefficient of variation. 


\begin{tabular}{|c|c|c|c|c|}
\hline Analytes & uRW & RMSbias & uCref & ubias \\
\hline $\mathrm{CRP}(\mathrm{mg} / \mathrm{L})$ & 1.23 & 1.79 & 1.02 & 2.06 \\
\hline Ferritin (ug/L) & 1.11 & 24.39 & 0.75 & 24.40 \\
\hline Procalcitonin (ng/mL) & 1.09 & $\mathrm{NC}$ & NC & NC \\
\hline Troponin I (ng/L) & 1.47 & 24.18 & 1.23 & 24.21 \\
\hline $\mathrm{BNP}(\mathrm{pg} / \mathrm{mL})$ & 1.90 & 26.84 & 1.23 & 26.87 \\
\hline
\end{tabular}

BNP: Brain natriuretic peptide; CRP: C-reactive protein; NC: Not calculated; RMSbias: Root-mean-square bias calculated according to the external quality control mean; ubias: Standard uncertainty; uCref: Nominal uncertainty; uRw: Uncertainty of within-laboratory reproducibility.

6-month data obtained from the EQAS program were used. The root-mean-square (RMS) bias and nominal uncertainty values (uCref) were calculated according to the external quality control data (Table 2). RMS bias was calculated using the formula $\sqrt{\Sigma}$ bias (external quality control)2/the number of laboratories participating in the external quality control program. The uCref was calculated by dividing the mean $\mathrm{CV}$ value (sR) obtained from the external quality control data by the square root of the mean number of laboratories using the same method and the same device $(s R / \sqrt{ } n)$. Intra-laboratory reproducibility ( $u R W$ ) was calculated using data obtained from consecutive measurement results of sample pools of different concentrations. Standard uncertainty (ubias) and combined standard uncertainty (u) were calculated using the formulas RMSbias ${ }^{2}+u \mathrm{Cref}^{2}$ and $\sqrt{ }(\mathrm{uR}-$ $\mathrm{W}^{2}+$ ubias $\left.^{2}\right) / 2$, respectively. Expanded uncertainty $(\mathrm{U})$ values were obtained by multiplying $u$ by a coverage factor $(k)$. The k-value was 1.96 for a $95 \%$ confidence interval.

The MU of the procalcitonin test could not be calculated due to insufficient external quality control data. The laboratory had just begun to implement this measurement as a result of the COVID-19 pandemic.

\section{Results}

The ubias value of CRP, ferritin, troponin I, and BNP tests was calculated to be $2.06,24.40,24.21$, and 26.87 , respectively. The MU of the CRP test was between $4.70 \%$ and $5.49 \%$. The values of uRW, RMSbias, uCref, and ubias calculated to determine the MU are presented in Table 2. The lowest MU values of ferritin, troponin I, and BNP were $48.45 \%, 48.24 \%$, and $53 \%$, respectively. The combined RCV value was $94.6 \%, 38.31 \%, 41.57 \%$, $42.35 \%$, and $29.36 \%$ for CRP, ferritin, procalcitonin, troponin $\mathrm{I}$, and BNP, respectively. The lowest RCV value of CRP, ferritin, procalcitonin, BNP, and troponin I tests was $94.57 \%, 37.16 \%$, $40.55 \%, 42.01 \%$, and $28.99 \%$, respectively. The calculated mean, $S D, C V_{a^{\prime}} R C V$, $u$, and $U$ values for different concentrations of CRP, ferritin, procalcitonin, troponin I, and BNP are presented in Table 3.

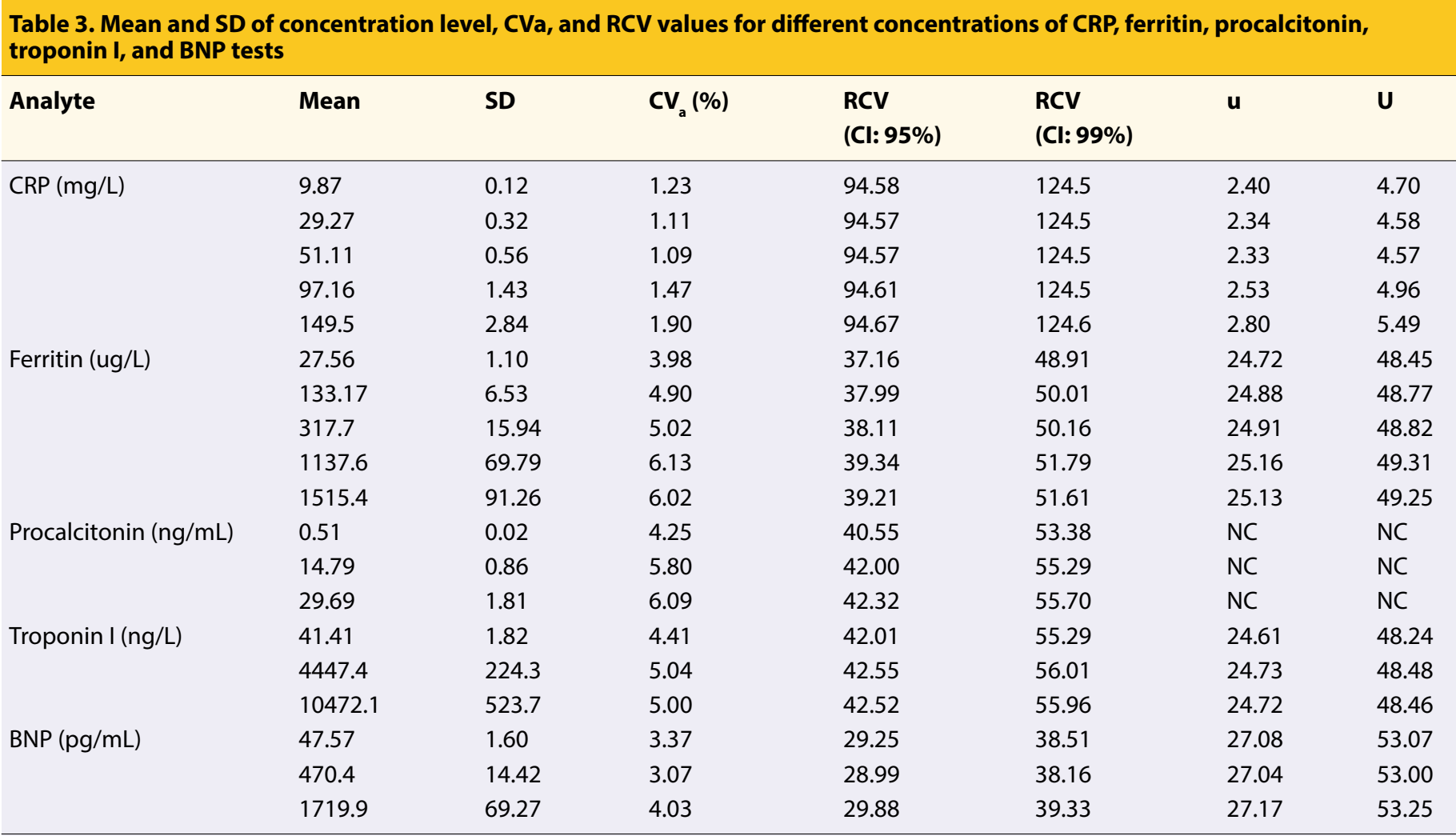

BNP: Brain natriuretic peptide; CRP: C-reactive protein; $\mathrm{CV}_{\mathrm{a}}$ : Analytical coefficient of variation; NC: Not calculated; RCV: Reference change value; U: Expanded uncertainty; u: Combined standard uncertainty. 
Table 4. The lower and upper limits of the measured quantity concentration according to the calculated RCV and MU values

\begin{tabular}{|c|c|c|c|c|c|}
\hline Analyte & Mean & \multicolumn{2}{|c|}{$\operatorname{RCV}(p<0.05)$} & \multicolumn{2}{|c|}{ Expanded MU } \\
\hline & 29.27 & 1.59 & 56.94 & 27.92 & 30.61 \\
\hline & 51.11 & 2.78 & 99.44 & 48.77 & 53.44 \\
\hline & 97.16 & 5.24 & 189.1 & 92.34 & 102.0 \\
\hline \multirow[t]{5}{*}{ Ferritin (ug/L) } & 27.56 & 17.32 & 37.8 & 14.21 & 40.91 \\
\hline & 133.2 & 82.58 & 183.8 & 68.22 & 198.1 \\
\hline & 317.7 & 196.6 & 438.7 & 162.6 & 472.8 \\
\hline & 1137.6 & 690.1 & 1585.1 & 576.6 & 1698.5 \\
\hline & 1515.4 & 921.2 & 2109.6 & 769.1 & 2261.7 \\
\hline & 4447.4 & 2555.0 & 6339.8 & 2291.3 & 6603.5 \\
\hline & 10472 & 6019.4 & 14924.9 & 5397.3 & 15546.9 \\
\hline \multirow[t]{3}{*}{$\mathrm{BNP}(\mathrm{pg} / \mathrm{mL})$} & 47.57 & 33.65 & 61.48 & 22.32 & 72.81 \\
\hline & 470.4 & 334.0 & 606.7 & 221.1 & 719.7 \\
\hline & 1719.9 & 1206.0 & 2233.9 & 804.1 & 2635.8 \\
\hline
\end{tabular}

BNP: Brain natriuretic peptide; CRP: C-reactive protein; MU: Measurement uncertainty; NC: Not calculated; RCV: Reference change value.

\section{Discussion}

Biochemical markers provide clinicians with valuable information to better predict COVID-19 severity and mortality, in addition to clinical, immunological, hematological, and radiographic findings. The CRP, ferritin, procalcitonin, troponin I, and BNP tests that we evaluated in this study are used as markers of disease severity in patients with COVID-19 [2,3,14]. Clinicians refer to these biochemical markers when making important decisions, such as hospitalization and treatment options. Concentrations of these analytes are monitored using sequential measurements in patients diagnosed with COVID-19. The ability to provide additional information about the characteristics of the measurement procedure along with the test results in laboratory reports provides valuable assistance to the correct interpretation of patient clinical status and the evaluation of response to treatment [15].

The allowable total error values (\%) of CRP, ferritin, troponin, BNP, and procalcitonin measurement procedures are $56.6,16.9,27.91,30$, and 20.3 , respectively [10, 11, 16]. In our study, we determined that the calculated $\mathrm{CV}_{\mathrm{a}}$ values of the CRP, ferritin, troponin, BNP, and procalcitonin measurement procedures were lower than the allowable total error values (Table 1). The analytical CV values calculated for different concentrations of the CRP test in our study revealed that the RCV value was $94.58-94.67 \%$ with a $95 \%$ confidence interval, and the combined RCV value was $94.6 \%$. The uncertainty value of the CRP test was between $4.70 \%$ and $5.49 \%$. Ac- cording to our data of consecutive measurements of CRP, it appears that the difference is clinically significant when the CRP test concentration changes approximately 2 times. The $R C V$ value is calculated using imprecision and $\mathrm{CV}_{i}$ data [12]. In tests where changes in sequential measurement results are monitored, the bias of the measurement procedure is not included in the RCV calculation, as it affects both test results measured, and therefore does not affect the difference between 2 sequential measurements. The circumstances are different when clinical decision points are used to evaluate test results. Since a single test result is important for clinical interpretation, it becomes important to evaluate the bias of the measurement procedure in addition to analytical variation. Therefore, it is valuable to determine the MU and present it to clinicians so that they can interpret the information appropriately and accurately. In our study, we determined the RCV and $M U$ values by calculating the $C_{a}$ values of tests with a high concentration as well as the $\mathrm{CV}_{\mathrm{a}}$ values of the clinical decision concentrations. The concentration of these markers is typically high in COVID-19 patients, whereas the clinical decision levels are controlled according to internal quality control studies. We found that the $\mathrm{CV}_{\mathrm{a}}$ values calculated for different concentrations of CRP measurement were below the $\mathrm{CV}_{\mathrm{a}}$ values for the critical decision points provided by the manufacturer. According to the Rilibak quality guidelines, the $\mathrm{CV}_{\mathrm{a}}$ of the CRP measurement procedure meets the minimum requirement [17]. 
Macy et al. [18] and Fraser et al. [19] similarly reported an RCV value of the CRP test of $118 \%$ and $175 \%$, respectively $(p<0.05)$. The critical difference value in our study was lower because the $\mathrm{CV}_{\mathrm{a}}$ value of our laboratory is lower than the $\mathrm{CV}_{\mathrm{a}}$ values in these other studies. In contrast, in the European Biological Variation study published in 2019, it was decided not to calculate the RCV value for the CRP measurement procedure due to a skewed value distribution in the population. The skewed value distribution was due to a low concentration of CRP under basal conditions and a 10-20-fold increase in cases of even minor inflammatory attack [20]. The ISO 15189: 2012 states that the MU value is of practical value to clinicians when it is used to evaluate the significance of the difference in a patient value with a previous value of the same type or clinical decision value [15]. Therefore, in the interpretation of consecutive results of a CRP test, the $M U$ value can be used as well as the RCV value. However, the debate about the bias component in measurement uncertainty is ongoing. The Guide to the Expression of Uncertainty in Measurement stated that the MU should be calculated by minimizing the bias value with tools such as recalibration [21]. This is important to remember when the MU is used to interpret consecutive measurements, as bias can affect both measurements [22].

In our study, the effect of bias could not be eliminated when calculating the MU. Therefore, bias should be considered a type B measurement error. As in the European Biological Variation Study [20], we think that it would be useful to consider at least the analytical variation value when interpreting the clinically significant change in consecutive measurements in cases where the RCV cannot be used due to biological variation.

The tests we evaluate in our laboratory are performed in a single analyzer. It should be noted that it is not appropriate to use the RCV value when analyzers with different $\mathrm{CV}_{\mathrm{a}}$ or measurement procedures are used [23].

The lowest RCV values of ferritin, procalcitonin, BNP, and troponin I tests in this study were $37.16 \%, 40.55 \%, 42.01 \%$ and $28.99 \%$, respectively. While the MU value of the procalcitonin

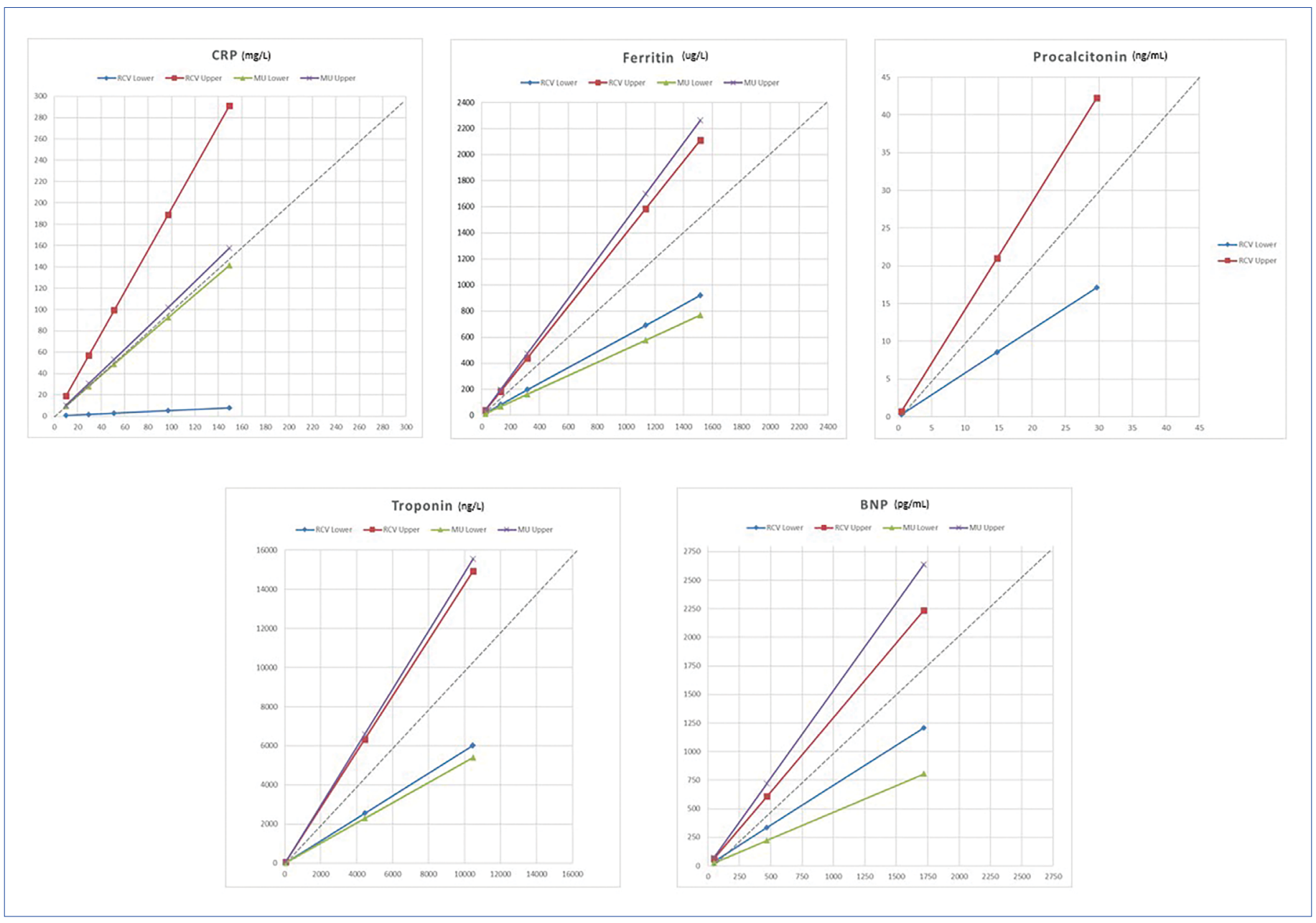

Figure 1. Upper and lower measurement uncertainty and reference change values of CRP, ferritin, procalcitonin, troponin I, and BNP tests according to concentration.

BNP: Brain natriuretic peptide; CRP: C-reactive protein; MU: Measurement uncertainty; RCV: Reference change value. 
measurement method could not be calculated due to insufficient external quality control result data, the lowest MU values of ferritin, troponin I, and BNP were calculated as $48.45 \%$, $48.24 \%$, and $53 \%$, respectively. It is evident that as the concentration of the tests increased, the numerical value of the significant clinical change and the attributed result range increased considerably (Table 4 and Fig.1). In high concentration values, the probability of erroneous interpretation may increase without considering clinically significant change value and MU data. Considering that the experience and intuition of clinicians are still part of the basic approach in patient care and monitoring of changes in sequential measurements, we think that the results of this study will contribute to important awareness of this issue [23].

Although the potential advantages and disadvantages of reporting the MU in medical laboratory reports are still a topic of discussion, the potential benefit of $\mathrm{MU}$ in clinical decisionmaking was noted in ISO 15189: 2012 [15].

COVID-19 is a new disease. Since data on the calculated CV $_{i}$ values for analytes in this patient group are not yet available, $\mathrm{CV}_{\mathrm{i}}$ values obtained from the general population were used in our study. This is a limitation of our research.

\section{Conclusion}

In conclusion, our findings indicate that the results of the tests we studies will be more useful in clinical evaluation when interpreted with the benefit of the RCV and MU values. This is particularly true for results at higher concentrations. Providing the RCV and MU values in addition to the test results in laboratory reports of these markers, which are frequently in high concentrations in COVID-19 patients, may be beneficial to more accurate clinical interpretation.

Conflict of Interest: The authors declare no conflict of interest.

Ethics Committee Approval: Not applicable.

Financial Disclosure: The authors have nothing to disclose.

Peer-review: Externally peer-reviewed.

Authorship Contributions: Concept - N.Z., O.Z.; Design - N.Z., O.Z.; Supervision - N.Z., O.Z.; Funding - N.Z., O.Z.; Materials - N.Z.; Data collection \&/or processing - N.Z.; Analysis and/or interpretation - N.Z., O.Z.; Literature search - N.Z., O.Z.; Writing - N.Z., O.Z;; Critical review - N.Z., O.Z.

\section{References}

1. Sharifpour M, Rangaraju S, Liu M, Alabyad D, Nahab FB, Creel-Bulos CM, et al. C-Reactive protein as a prognostic indicator in hospitalized patients with COVID-19. PLoS One 2020;15(11):e0242400. [CrossRef]

2. Velavan TP, Meyer CG. Mild versus severe COVID-19: Laboratory markers. Int J Infect Dis 2020;95:304-7. [CrossRef]
3. Tan L, Kang X, Ji X, Li G, Wang Q, Li Y, et al. Validation of predictors of disease severity and outcomes in COVID-19 patients: a descriptive and retrospective study. Med (N Y) 2020;1(1):12838.e3. [CrossRef]

4. Chen W, Zheng KI, Liu S, Yan Z, Xu C, Qiao Z. Plasma CRP level is positively associated with the severity of COVID-19. Ann Clin Microbiol Antimicrob 2020;19(1):18. [CrossRef]

5. Dallas Jones GR. Measurement uncertainty for clinical laboratories - a revision of the concept. Clin Chem Lab Med 2016;54(8):1303-7. [CrossRef]

6. Harris EK, Brown SS. Temporal changes in the concentrations of serum constituents in healthy men. Distributions of within-person variances and their relevance to the interpretation of differences between successive measurements. Ann Clin Biochem 1979;16(4):169-76. [CrossRef]

7. Harris EK, Yasaka T. On the calculation of a "reference change" for comparing two consecutive measurements. Clin Chem 1983;29(1):25-30. [CrossRef]

8. Ćelap I, Vukasović I, Juričić G, Šimundić AM. Minimum requirements for the estimation of measurement uncertainty: Recommendations of the joint Working group for uncertainty of measurement of the CSMBLM and CCMB. Biochem Med (Zagreb) 2017;27(3):030502. [CrossRef]

9. Aarsand AK, Fernandez-Calle P, Webster C, Coskun A, Gonzales-Lao E, Diaz-Garzon J, et al. The EFLM biological variation database. Available at: https://biologicalvariation.eu/. Accessed Jan 20, 2021.

10. Barassi A, Pallotti F, Melzi d'Eril G. Biological variation of procalcitonin in healthy individuals. Clin Chem 2004;50(10):1878.

11. Westgard J. CV individual. Available at: https://www.westgard. com/biodatabase1.htm. Accessed Jan 20, 2021. [CrossRef]

12. Fraser CG. Reference change values. Clin Chem Lab Med 2011;50(5):807-12. [CrossRef]

13. Magnusson B, Naykki T, Hovind H, Krysell M. Handbook for calculation of measurement uncertainty in 202 environmental laboratories, NordTest Report TR 537. Finland: NordTest; 2004.

14. Kavsak PA, Hammarsten O, Worster A, Smith SW, Apple FS. Cardiac troponin testing in patients with COVID-19: a strategy for testing and reporting results. Clin Chem 2021;67(1):107-13.

15. ISO 15189, Medical laboratories - Particular requirements for quality and competence. International Organization for Standardization (ISO) 15189. Geneva: ISO; 2012.

16. Westgard J. 2019: CLIA proposed changes to PT acceptable limits. Available at: https://www.westgard.com/2019-cliachanges.htm. Accessed Jul 20, 2021.

17. Westgard QC. Rilibak: quality goals the German way. Available at: http://www.westgard.com/rilibak-2.htm. Accessed Jan 20, 2021.

18. Macy EM, Hayes TE, Tracy RP. Variability in the measurement of C-reactive protein in healthy subjects: implications for reference intervals and epidemiological applications. Clin Chem 1997;43(1):52-8. [CrossRef]

19. Clark GH, Fraser CG. Biological variation of acute phase proteins. Ann Clin Biochem 1993;30:373-6. [CrossRef] 
20. Carobene A, Aarsand AK, Guerra E, Bartlett WA, Coşkun A, DíazGarzón J, et al; European Federation of Clinical Chemistry and Laboratory Medicine Working Group on Biological Variation. European Biological Variation Study (EuBIVAS): within- and between-subject biological variation data for 15 frequently measured proteins. Clin Chem 2019;65(8):1031-41. [CrossRef]

21. JCGM 100:2008, Evaluation of measurement data - guide to the expression of uncertainty in measurement (GUM). Avail- able at: https://www.bipm.org/utils/common/documents/ jcgm/JCGM_100_2008_E.pdf. Accessed Jan 20, 2021.

22. Padoan A, Sciacovelli L, Aita A, Antonelli G, Plebani M. Measurement uncertainty in laboratory reports: $A$ tool for improving the interpretation of test results. Clin Biochem 2018;57:41-7.

23. Rigo-Bonnin R, Muñoz-Provencio D, Canalias F. Reference change values based on uncertainty models. Clin Biochem 2020;80:31-41. [CrossRef] 